\title{
Charge expulsion, charge inhomogeneity and phase separation in dynamic Hubbard models
}

\author{
J. E. Hirsch \\ Department of Physics, University of California, San Diego \\ La Jolla, CA 92093-0319
}

(Dated: July 11, 2019)

\begin{abstract}
Dynamic Hubbard models are extensions of the conventional Hubbard model that take into account the fact that atomic orbitals expand upon double occupancy. It is shown here that systems described by dynamic Hubbard models have a tendency to expel negative charge from their interior to the surface, and to develop charge inhomogeneity and even phase separation in the bulk. These effects are associated with lowering of electronic kinetic energy. We propose that these models may explain the charge inhomogeneity and negatively charged grain boundaries observed in cuprate oxides and other materials.
\end{abstract}

PACS numbers:

\section{INTRODUCTION}

The conventional single band Hubbard model with Hamiltonian

$$
H=-\sum_{i, j, \sigma}\left[t_{i j} c_{i \sigma}^{\dagger} c_{j \sigma}+\text { h.c. }\right]+U \sum_{i} n_{i \uparrow} n_{i \downarrow}
$$

has been used to describe the physics of many real materials [1, 2]. The model ignores the fact that nondegenerate atomic orbitals are necessarily modified by double electronic occupancy $3[6]$. To remedy this deficiency a variety of new Hamiltonians have been proposed and studied that we will generically call 'dynamic Hubbard models', that take into account the fact that orbital expansion takes place when a non-degenerate atomic orbital is doubly occupied [7-17].

The essential physics of real atoms that is described by dynamic Hubbard models but not by the conventional Hubbard model is shown in Fig. 1: when a second electron (of opposite spin) is added to a non-degenerate orbital, it expands, due to electron-electron repulsion. This has two key consequences at the atomic level. One is, negative charge moves outward. The second is, the kinetic energy of the electrons is lowered: in an orbital of radial extent $r$ the electron kinetic energy is of order $\hbar^{2} /\left(2 m_{e} r^{2}\right)$, with $m_{e}$ the electron mass. The kinetic energy is lowered since the expanded orbital has larger radius than the original one. Remarkably, we will find that these properties at the atomic level, negative charge expulsion and kinetic energy lowering, are also reflected in the properties of dynamic Hubbard models at the macroscopic level. At the local level, effects described by the dynamic Hubbard model that are not described by the conventional Hubbard model can be experimentally probed by ultrafast quantum modulation spectroscopy as recently demonstrated[18].

One of several [19] ways to incorporate this physics in the Hubbard Hamiltonian is by the substitution [9, 20, 21]

$$
U n_{i \uparrow} n_{i \downarrow} \rightarrow\left(U+\alpha q_{i}\right) n_{i \uparrow} n_{i \downarrow}
$$

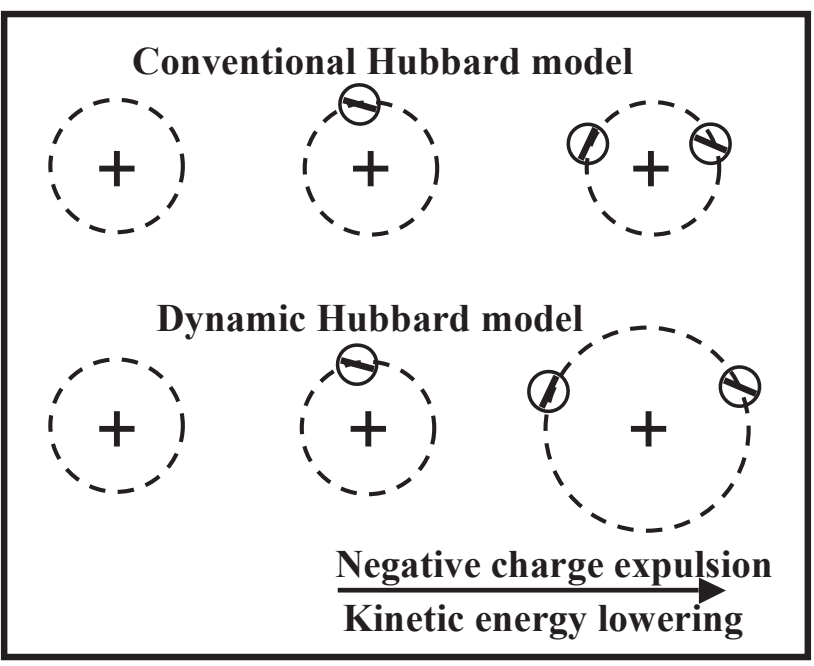

FIG. 1: In the conventional Hubbard model the atomic orbital is not modified by electronic occupancy. In the dynamic Hubbard model and in real atoms, addition of the second electron causes orbital expansion due to the electron-electron interaction. Negative charge is expelled outward and the kinetic energy of the electrons is lowered relative to that with a non-expanded orbital.

where $\alpha$ is a coupling constant (assumed positive) and $q_{i}$ a local boson degree of freedom describing the orbital relaxation, with equilibrium position at $q_{i}=0$ if zero or one electrons are present: upon double occupancy of the orbital at site $i, q_{i}$ will change from zero to a negative value to reduce the electronic on-site repulsion, to an extent determined by the boson dynamics. If we describe the dynamics of this boson by a simple harmonic oscillator 20]

$$
H_{i}=\frac{p_{i}^{2}}{2 m}+\frac{1}{2} K q_{i}^{2}+\left(U+\alpha q_{i}\right) n_{i \uparrow} n_{i \downarrow}
$$

the on-site repulsion is reduced from $U$ to $U_{\text {eff }}=U-$ $\alpha^{2} /(2 K)$ when $q_{i}$ takes the value $q_{i}=-\alpha / K$ corresponding to minimum energy when the site is doubly occupied, 
versus $q_{i}=0$ when the site is unoccupied or singly occupied. The conventional Hubbard model does not allow the orbital to relax, in other words it corresponds to the limit $K \rightarrow \infty$ of an infinitely stiff orbital.

Superficially the site Hamiltonian Eq. (3) may look like the conventional Holstein model giving rise to small polarons but it is in fact very different: the boson degree of freedom $q_{i}$ couples to the double occupancy of the site $\left(n_{i \uparrow} n_{i \downarrow}\right)$ rather than to the single occupancy $\left(n_{i \uparrow}+n_{i \downarrow}\right)$. Such a coupling, in a context where $q_{i}$ represented a phonon degree of freedom, was first introduced by Pincus [20] but not often considered thereafter.

The Hamiltonian Eq. (3) is intrinsically electron-hole asymmetric [9]: the importance of this physics increases as the filling of the electronic energy band increases, as is simply seen by taking the mean field expectation value of Eq. (3). In addition, the importance of this physics increases when the ionic charge is small[9], since in that case the orbital expansion is larger (for example, the orbital expansion is larger for $\mathrm{H}^{-}$than for $\mathrm{He}$ ), corresponding to a smaller stiffness parameter $K$ in Eq. (3). These two facts imply that the importance of this physics increases the more negative charge the system has. Notably, we will find in this paper that systems described by this Hamiltonian have a strong tendency to expel negative charge, reflecting the radial expulsion of negative charge that already exists at the atomic level. Similarly, we will find for the system as a whole described by a dynamic Hubbard model that negative charge expulsion is associated with lowering of kinetic energy, just like in the atom. Note however that our site Hamiltonian Eq. (3), describing orbital expansion when the value of $q_{i}$ is negative, does not have a term explicitly describing the kinetic energy lowering of the atomic electron when $q_{i}$ adopts a negative value.

The potential (Coulomb) energy of a system of charges is minimized when the charge distribution is uniform. A non-uniform charge distribution gives rise to electrostatic fields and an associated potential energy cost. It will be favored if this cost is compensated by a kinetic energy gain, i.e. lowering of kinetic energy. In dynamic Hubbard models kinetic energy plays a key role, and we will find in this paper that dynamic Hubbard models are prone to develop charge inhomogeneity, and in extreme cases charge separation, where kinetic energy lowering overcompensates for the potential energy cost. Many materials of recent interest, including high $T_{c}$ cuprates and manganites, exhibit charge inhomogeneity [22 26], suggesting that dynamic Hubbard models may be useful to describe them. In addition, the tendency to charge inhomogeneity will influence the charge distribution around grain boundaries, as we discuss in this paper.

One may ask whether dynamic Hubbard models are really fundamentally different from many other models proposed and extensively studied in the literature such as the conventional Hubbard model, the periodic Anderson model, the Falicov-Kimball model, the Holstein model, the Holstein-Hubbard model, the Fröhlich Hamiltonian, standard polaron models, standard electron-boson models, the t-J model, etc, so as to merit a new name and new studies. We believe most definitely yes. These conventional models are usually assumed to be electron-hole symmetric, or at least electron-hole asymmetry does not play a key role, and the interesting physics in these models is usually driven by electronic correlation and potential energy rather than by kinetic energy. Instead, the essential physics of dynamic Hubbard models is electronhole asymmetry, and the physics is kinetic energy driven. On the other hand some of these conventional models mentioned above, when suitably modified, have properties in common with dynamic Hubbard models. In particular, electron-hole asymmetric polaron models [27] arising from coupling to either electronic or spin or phononic degrees of freedom in ways that break electron-hole symmetry have much in common with the model discussed here.

Finally, it is certainly possible that some the physics of dynamic Hubbard models is also contained in some models studied in the past under a different name. For example, Robaszkiewicz et al[28] studied a generalized periodic Anderson model with a wide and a narrow band, with 10 different pieces to their initial Hamiltonian (Eq. (2.1)). After a Lang-Firsov transformation leading to a small polaron representation they end up with a Hamiltonian (Eq. (2.10)) that has 18 different terms (Eq. 2.11), one of which bears some resemblance to a term in our Hamiltonian (which has 3 terms rather than 18). As a consequence, the focus of that paper is very different from the physics discussed here.

\section{DYNAMIC HUBBARD MODELS}

We can describe the physics depicted in Fig. 1 by a two-orbital tight binding model (for the unexpanded and expanded orbital) [8, 29], or with a background spin [4, 21] or harmonic oscillator [30, 31] degree of freedom that is coupled to the electronic double occupancy, as in Eq. (2). We expect the physics to be similar for all these cases. Assuming the latter, the site Hamiltonian is given by Eq. (3), and the Hamiltonian can be written as

$$
\begin{aligned}
H & =-\sum_{i, j, \sigma}\left[t_{i j} c_{i \sigma}^{\dagger} c_{j \sigma}+h . c .\right]+\sum_{i} \hbar \omega_{0} a_{i}^{\dagger} a_{i} \\
& +\sum_{i}\left[U+g \hbar \omega_{0}\left(a_{i}^{\dagger}+a_{i}\right)\right] n_{i \uparrow} n_{i \downarrow}
\end{aligned}
$$

with frequency $\omega_{0}=\sqrt{K / m}$ and $g=\alpha /\left(2 K \hbar \omega_{0}\right)^{1 / 2}$ the dimensionless coupling constant. Estimates for the values of these parameters were discussed in ref. [9]. In particular, for $1 s$ orbitals $g^{2} \hbar \omega_{0} \sim 4.1 \mathrm{eV}$. Quite generally we expect $g$ to increase proportionally to $1 / Z$ and $\omega_{0}$ to increase proportionally to $Z^{2}$, where $Z$ is the charge of the ion when the orbital under consideration is empty [9]. However, in an even more realistic model $\omega_{0}$ should also 
change with different electronic occupation. That issue is beyond the scope of this paper.

Using a generalized Lang-Firsov transformation [31, 32] the electron creation operator $c_{i \sigma}^{\dagger}$ is written in terms of new quasiparticle operators $\tilde{c}_{i \sigma}^{\dagger}$ as

$$
\begin{aligned}
c_{i \sigma}^{\dagger} & =e^{g\left(a_{i}^{\dagger}-a_{i}\right) \tilde{n}_{i,-\sigma}} \tilde{c}_{i \sigma}^{\dagger}=\left[1+\left(e^{-g^{2} / 2}-1\right) \tilde{n}_{i,-\sigma}\right] \tilde{c}_{i \sigma}^{\dagger} \\
& +\tilde{n}_{i,-\sigma} \times(\text { incoherent part })
\end{aligned}
$$

where the incoherent part describes the processes where the boson goes into an excited state when the electron is created at the site. For large $\omega_{0}$ those terms become small and can be neglected, and even for not so large $\omega_{0}$ we have found from numerical studies that their effect does not change the low energy physics qualitatively [33, 34]. Hence we will ignore those terms in what follows, which amounts to keeping only ground state to ground state transitions of the boson field.

The electron creation operator is then given by

$$
\begin{gathered}
c_{i \sigma}^{\dagger}=\left[1+(S-1) \tilde{n}_{i,-\sigma}\right] \tilde{c}_{i \sigma}^{\dagger} \\
S=e^{-g^{2} / 2}
\end{gathered}
$$

and the quasiparticle weight for electronic band filling $n$ ( $n$ electrons per site) is

$$
z(n)=\left(1+(S-1) \frac{n}{2}\right)^{2}
$$

so that it decreases monotonically from 1 when the band is almost empty to $S^{2}<1$ when the band is almost full. The single particle Green's function and associated spectral function is renormalized by the multiplicative factors on the quasiparticle operators given in Eq. (6a)) 31], which on the average amounts to multiplication of the spectral function by the quasiparticle weight Eq. (6c). This will cause a reduction in the photoemission spectral weight at low energies from what would naively follow from the low energy effective Hamiltonian, an effect extensively discussed in Ref. 31] and recently rediscovered in 35]. A corresponding reduction occurs in the twoparticle Green's function and associated low frequency optical properties [31, 33].

According to Eq. $(6)<c_{i \sigma}^{\dagger} c_{i \sigma}>=z(n)<\tilde{c}_{i \sigma}^{\dagger} \tilde{c}_{i \sigma}>$, which appears to indicate that quasiparticles carry a different charge than real particles. This is however not the case, as can be seen by using Eq. (5) instead of Eq. (6) to evaluate $<\tilde{c}_{i \sigma}^{\dagger} \tilde{c}_{i \sigma}>$. The incoherent part accounts for the difference and in fact the quasiparticle carries an unrenormalized unit charge equal to that of the real particle, just as in usual Landau theory [36]. Therefore, we can obtain the real charge distribution in the system by computing the site occupations of the quasiparticles.

The low energy effective Hamiltonian is then

$$
H=-\sum_{i j \sigma} t_{i j}^{\sigma}\left[\tilde{c}_{i \sigma}^{\dagger} \tilde{c}_{j \sigma}+\text { h.c. }\right]+U_{e f f} \sum_{i} \tilde{n}_{i \uparrow} \tilde{n}_{i \downarrow}
$$

$$
t_{i j}^{\sigma}=\left[1+(S-1) \tilde{n}_{i,-\sigma}\right]\left[1+(S-1) \tilde{n}_{j,-\sigma}\right] t_{i j}
$$

and $U_{\text {eff }}=U-\hbar \omega_{0} g^{2}$. Thus, the hopping amplitude for an electron between sites $i$ and $j$ is given by $t_{i j}, S t_{i j}$ and $S^{2} t_{i j}$ depending on whether there are 0,1 or 2 other electrons of opposite spin at the two sites involved in the hopping process.

The physics of these models is determined by the magnitude of the parameter $S$, which can be understood as the overlap matrix element between the expanded and unexpanded orbital in Fig. 1. It depends crucially on the net ionic charge $Z$, defined as the ionic charge when the orbital in question is unoccupied [9]. In Fig. $1, Z=1$ if the states depicted correspond to the hydrogen ions $H^{+}, H$ and $H^{-}$and $Z=2$ if they correspond to $\mathrm{He}^{++}$, $\mathrm{He}^{+}$and $\mathrm{He}$. In a lattice of $\mathrm{O}^{=}$anions, as in the $\mathrm{Cu}-\mathrm{O}$ planes of high $T_{c}$ cuprates, the states under consideration are $O, O^{-}$and $O^{=}$and $Z=0$, and in the $B^{-}$planes of $M g B_{2}, Z=1$. The effects under consideration here become larger when $S$ is small, hence when $Z$ is small. An approximate calculation of $S$ as a function of $Z$ is given in [9].

We now perform a particle-hole transformation since we will be interested in the regime of low hole concentration. We assume for simplicity that the Hamiltonian is defined on a hypercubic lattice with only nearest neighbor hopping, so that the particle-hole transformation leaves the first term in the Hamiltonian Eq. (7a) unchanged by a suitable transformation of the phases. The hole creation operator is given by, instead of Eq. (6a)

$$
c_{i \sigma}^{\dagger}=\left[S+(1-S) \tilde{n}_{i,-\sigma}\right] \tilde{c}_{i \sigma}^{\dagger}
$$

where $\tilde{n}_{i, \sigma}$ is now the hole site occupation, and the hole quasiparticle weight increases with hole occupation $n$ as

$$
z_{h}(n)=S^{2}\left(1+\left(\frac{1}{S}-1\right) \frac{n}{2}\right)^{2}
$$

For simplicity of notation we denote the hole creation operators again by $c_{i \sigma}^{\dagger}$, the hole site occupation by $n_{i \sigma}$ and the effective on-site repulsion between holes of opposite spin $U_{\text {eff }}$ (the same as between electrons) by $U$ to simplify the notation. The Hamiltonian for holes is then

$$
\begin{gathered}
H=-\sum_{i j \sigma} t_{i j}^{\sigma}\left[c_{i \sigma}^{\dagger} c_{j \sigma}+\text { h.c. }\right]+U \sum_{i} n_{i \uparrow} n_{i \downarrow} \\
\left.t_{i j}^{\sigma}=t_{i j}^{h}\left[1+\left(\frac{1}{S}-1\right)\right) n_{i,-\sigma}\right]\left[1+\left(\frac{1}{S}-1\right) n_{j,-\sigma}\right] t_{i j}
\end{gathered}
$$

with $t_{i j}^{h}=S^{2} t_{i j}$ the hopping amplitude for a single hole when there are no other holes in the two sites involved in the hopping process. The hole hopping amplitude and the effective bandwidth increase as the hole occupation increases, and so does the quasiparticle (quasihole) weight Eq. (8b).

Assuming there is only nearest neighbor hopping $t_{i j}=$ $t$, the nearest neighbor hopping amplitude resulting from Eq. (9b) is

$$
t_{i j}^{\sigma}=t_{h}+\Delta t\left(n_{i,-\sigma}+n_{j,-\sigma}\right)+\Delta t_{2} n_{i,-\sigma} n_{j,-\sigma}
$$


with

$$
\begin{gathered}
t_{h}=t S^{2} \\
\Delta t=t S(1-S) \\
\Delta t_{2}=t(1-S)^{2}=(\Delta t)^{2} / t_{h} .
\end{gathered}
$$

The non-linear term with coefficient $\Delta t_{2}$ is expected to have a small effect when the band is close to full (with electrons) and is often neglected. Without that term, the model is also called the generalized Hubbard model or Hubbard model with correlated hopping 37, 38]. The effective hopping amplitude for average site occupation $n$ is, from Eq. (10a)

$$
t(n)=t_{h}+n \Delta t+\frac{n^{2}}{4} \Delta t_{2}
$$

so that a key consequence of integrating out the higher energy degrees of freedom is to renormalize the hopping amplitude and hence the bandwidth and the effective mass (inverse of hopping amplitude), as recently rediscovered in a different context 35$]$.

\section{GENERALIZED DYNAMIC HUBBARD MODELS}

More generally, one can assume that the boson degree of freedom will couple not only to the double orbital occupancy but also to the singly occupied orbital [31]. The site Hamiltonian is then

$$
H_{i}=\hbar \omega_{0} a_{i}^{\dagger} a_{i}+\hbar \omega_{0}\left(a_{i}^{\dagger}+a_{i}\right)\left[g n_{i \uparrow} n_{i \downarrow}+g_{0}\left(n_{i \uparrow}+n_{i \downarrow}\right)\right]
$$

At the atomic level, the coupling $g_{0}$ will appear when considering an orbital for atoms other than hydrogenic ones, and represents the modification of the states of electrons in other orbitals in the atom when the first electron is created in the orbital under consideration. We expect this effect to be much smaller than the modification of the state of the electron residing in the same orbital, the physics described by $g$, hence $g_{0}<<g$, particularly when the ionic charge $Z$ is small. The formal development for the site Hamiltonian given by Eq. (12) is very similar to the one discussed in the previous section and is given in Ref. 31]. In particular, Eq. (6) becomes

$$
\begin{gathered}
c_{i \sigma}^{\dagger}=\left[T+(S-T) \tilde{n}_{i,-\sigma}\right] \tilde{c}_{i \sigma}^{\dagger} \\
T=e^{-g_{0}^{2} / 2} \\
S=e^{-\left(g+g_{0}\right)^{2} / 2} \\
z(n)=\left(T+(S-T) \frac{n}{2}\right)^{2} .
\end{gathered}
$$

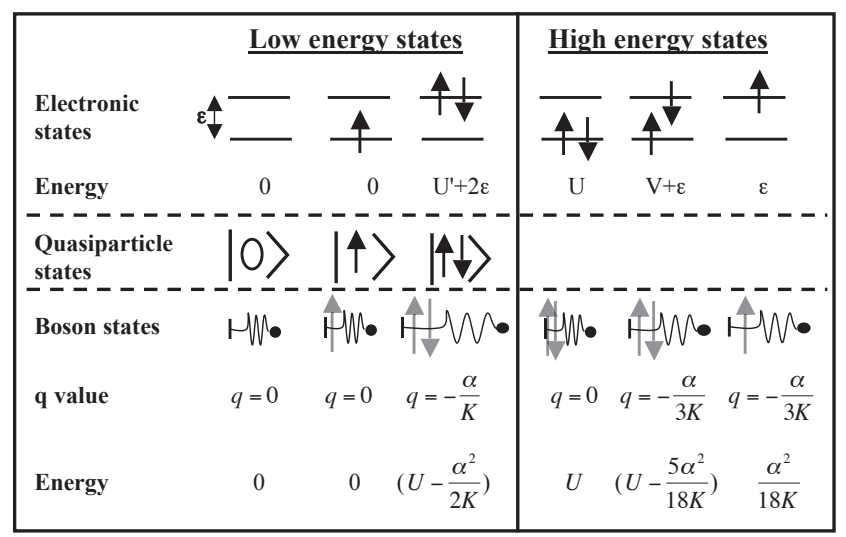

FIG. 2: Correspondence between states in the electronic dynamic Hubbard model introduced in ref. [8] (upper part of the figure) and the bosonic dynamic Hubbard model discussed here (lower part of the figure). Only a few representative high energy states are shown.

and of course $S<T$ always [21] since $g>0$. The hopping amplitudes given in the previous section are similarly modified by replacing 1 by $T$ in various places, as discussed in Ref. [31]. $g_{0}$ gives a renormalization of the quasiparticle mass, bandwidth and quasiparticle weight that is independent of band filling, and $g$ gives a bandfilling dependent contribution.

Recently, a single band model with site Hamiltonian of the form Eq. (12) with $g=0$ and $g_{0} \neq 0$ was considered [35] to describe the effect of higher energy electronic excitations on the low energy electronic physics within dynamical mean field theory [39]. In our view this is an unphysical limit since we expect $g>>g_{0}$ quite generally. Some of the effects discussed in refs. [9, 31, 33, 34] were rediscovered in that work 35$]$.

\section{ELECTRONIC VERSUS BOSONIC DYNAMIC HUBBARD MODELS}

Here we discuss briefly the relationship between the dynamic Hubbard model with site Hamiltonian given by Eq. (3) and the electronic model with two orbitals per site introduced in ref. [8] and discussed further in ref. [29], with site Hamiltonian

$$
H=U n_{\uparrow} n_{\downarrow}+U^{\prime} n_{\uparrow}^{\prime} n_{\downarrow}^{\prime}+V n n^{\prime}+\epsilon n^{\prime}-t^{\prime}\left(c_{\sigma}^{\dagger} c_{\sigma}^{\prime}+\text { h.c. }\right)
$$

The unprimed and primed operators describe electrons in the lower and upper atomic orbitals, with single particle energy difference $\epsilon$. These orbitals represent the unexpanded and expanded orbitals depicted in Fig. 1.

Fig. 2 shows the correspondence between the site states of the electronic and bosonic models. Parameters in the electronic model are chosen so that when the second electron is introduced at the site, the energy of the state with both electrons occupying the upper electronic state $\left(U^{\prime}+2 \epsilon\right)$ is lower than both the energy of the 
state with both electrons in the lower state $(U)$ and the state with one electron in each of the site states $(V+\epsilon)$. Similarly, in the bosonic model the two electron state when the oscillator is fully relaxed $(q=-\alpha / K)$ has lower energy than the state where the oscillator is unrelaxed $(q=0)$ or partially relaxed $(q=-\alpha / 3 K)$.

The details of the high energy states in both models are different, in particular the bosonic model has an infinite number of high energy states and the electronic model only a finite number. However, the low energy effective Hamiltonian Eq. (7) is the same for both models, and as a consequence the charge expulsion physics discussed here is the same for both models. Furthermore the physics of spectral weight transfer from high to low energies (undressing) [31] is the same for both models. Therefore, we argue that the electronic two-orbital model with the constraints on the interaction parameters assumed [8] and the bosonic model are essentially equivalent realizations of the physics of dynamic Hubbard models.

\section{NEGATIVE CHARGE EXPULSION}

We consider the Hamiltonian for holes Eq. (9), with the hopping amplitudes given by Eq. (10), which we reproduce here for convenience:

$$
\begin{gathered}
H=-\sum_{i j \sigma} t_{i j}^{\sigma}\left[c_{i \sigma}^{\dagger} c_{j \sigma}+\text { h.c. }\right]+U \sum_{i} n_{i \uparrow} n_{i \downarrow} \\
t_{i j}^{\sigma}=t_{h}+\Delta t\left(n_{i,-\sigma}+n_{j,-\sigma}\right)+\Delta t_{2} n_{i,-\sigma} n_{j,-\sigma}
\end{gathered}
$$

It is clear from the form of this Hamiltonian that the kinetic energy decreases when the number of holes in the band increases, since the hopping amplitudes Eq. (14b) increase with hole occupation. This suggest that the system will have a tendency to expel electrons from its interior to the surface, because the coordination of sites in the interior is larger than of sites at the surface. In what follows we study this physics numerically.

We assume a cylindrical geometry of radius $\mathrm{R}$ and infinite length in the $\mathrm{z}$ direction. We decouple the interaction terms within a simple mean field approximation assuming $\left\langle n_{i \sigma}>=n_{i} / 2\right.$ with $n_{i}$ the hole occupation at site $i$, and obtain the mean field Hamiltonian

$$
\begin{gathered}
H_{m f}=H_{m f, k i n}+H_{m f, p o t}+H_{\mu} \\
H_{m f, k i n}=-\sum_{<i j>, \sigma}\left[t_{h}+\Delta t n_{i}+\Delta t_{2} \frac{n_{i}^{2}}{4}\right]\left[c_{i \sigma}^{\dagger} c_{j \sigma}+h . c .\right] \\
H_{m f, p o t}=\frac{U}{4} \sum_{i} n_{i}^{2} \\
H_{\mu}=-\sum_{<i j>} n_{i}\left[\Delta t+\frac{n_{j}}{2} \Delta t_{2}\right] \sum_{\sigma}<c_{i \sigma}^{\dagger} c_{j \sigma}>
\end{gathered}
$$

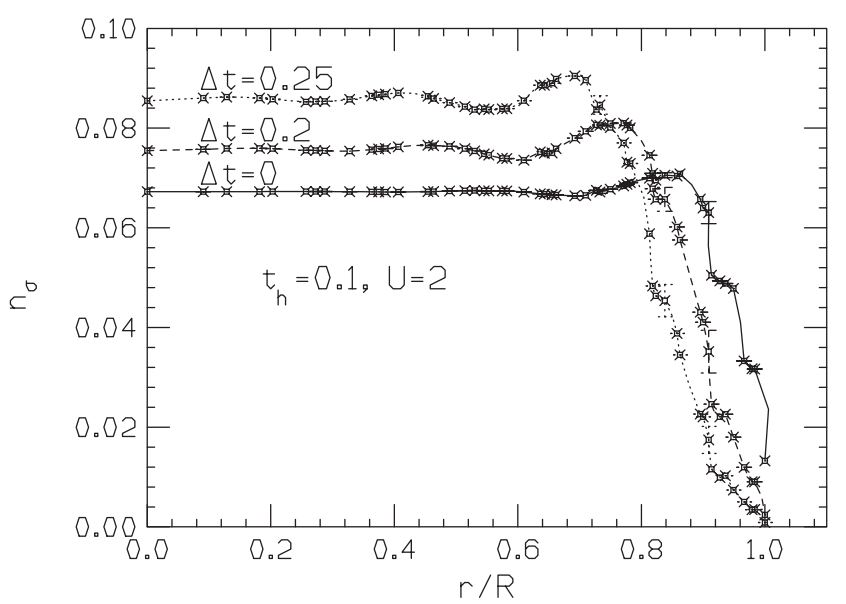

FIG. 3: Hole site occupation per spin for a cylinder of radius $R=11$ as function of $r / R$, with $r$ the distance to the center, for a cubic lattice of side length 1 . There are 377 sites in a cross-sectional area $\left(\pi R^{2}=380.1\right)$. The average occupation (both spins) is $n=0.126$ holes per site and the temperature is $k_{B} T=0.02$.

Note that the local average bond occupation modifies the local chemical potential. Assuming a band filling of $n$ holes per site, we diagonalize the Hamiltonian Eq. (15) with initial values $n_{i}=n$ and fill the lowest energy levels until the occupation $n$ is achieved. From the Slater determinant of that state we obtain new values of $n_{i}$ for each site and for the local bond occupation, and iterate this procedure until self-consistently is achieved. We can extend this procedure to finite temperatures, iterating to self-consistency for a given chemical potential $\mu$. We consider then the resulting occupation of the sites as function of the distance $r$ to the center of the cylinder. Sometimes there are non-equivalent sites at the same distance from the axis (e.g. $(5,0)$ and $(3,4))$ that yield somewhat different occupation, for those cases we show the average and standard deviation as error bars in the graphs.

Figure 3 shows a typical example of the behavior found. Here we assumed $\Delta t_{2}=0$, corresponding to the simpler Hubbard model with correlated hopping and no sixfermion operator term. Even for $\Delta t=0$ the hole occupation is somewhat larger in the interior than near the surface. When the interaction $\Delta t$ is turned on, the hole occupation increases in the interior and decreases near the surface. This indicates that the system expels electrons from the interior to the surface. The effect becomes more pronounced when $\Delta t$ is increased.

Figure 4 shows the hole site occupations as circles of diameter proportional to it, for the cases $\Delta t=0$ and $\Delta t=0.25$ of Fig. 3. Note that the interior hole occupation is larger for $\Delta t=0.25$ than it is for $\Delta t=0$, while near the surface the hole occupation is larger for $\Delta t=0$. Again this shows that the system with $\Delta t=0.25$ is expelling electrons from the interior to the surface, thus depleting the hole occupation near the surface.

These results are obtained by iteration. Fig. 5 shows 

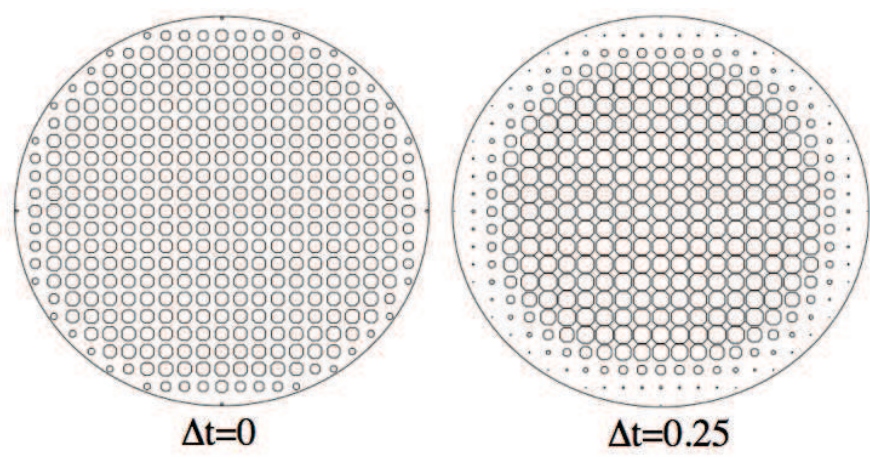

FIG. 4: The diameters of the circles are proportional to the hole occupation of the site. Note that for finite $\Delta t$ the hole occupation increases in the interior and is depleted near the surface. The parameters correspond to the cases shown in Fig. 3.

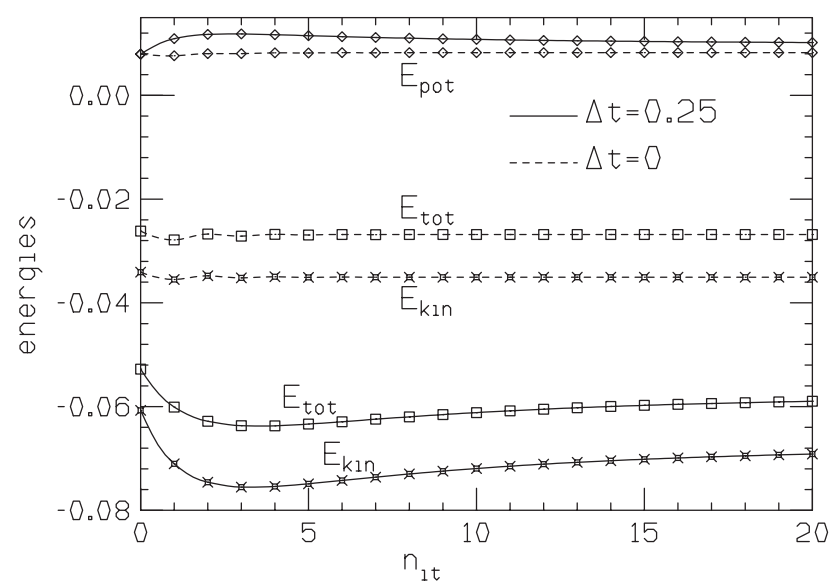

FIG. 5: Kinetic, potential and total energy per site for $\Delta t=0.25$ as function of number of iterations starting with a uniform hole distribution.

the behavior of the energies as a function of iteration number for the cases $\Delta t=0$ and $\Delta t=0.25$ of Fig. 3 . The initial values correspond to a uniform hole distribution with each site having the average occupation. The evolution is non-monotonic because in the intermediate steps the overall hole concentration increases, nevertheless it can be seen that for the case $\Delta t=0.25$ the final kinetic energy when self-consistency is achieved is lower, and the final potential energy is higher, associated with the larger hole concentration in the interior and the lower hole concentration near the surface shown in Fig. 4. This is of course what is expected. For the case $\Delta t=0$ instead there is essentially no difference in the energies between the initial uniform state and the final self-consistent state.

The charge expulsion caused by $\Delta t$ is counteracted by the effect of Coulomb repulsion. Figure 6 shows the effect of increasing the on-site repulsion for a fixed value of $\Delta t$.

The effect of the non-linear occupation-dependent hopping term $\Delta t_{2}$ (Eq. (10d)) is shown in Fig. 7. It

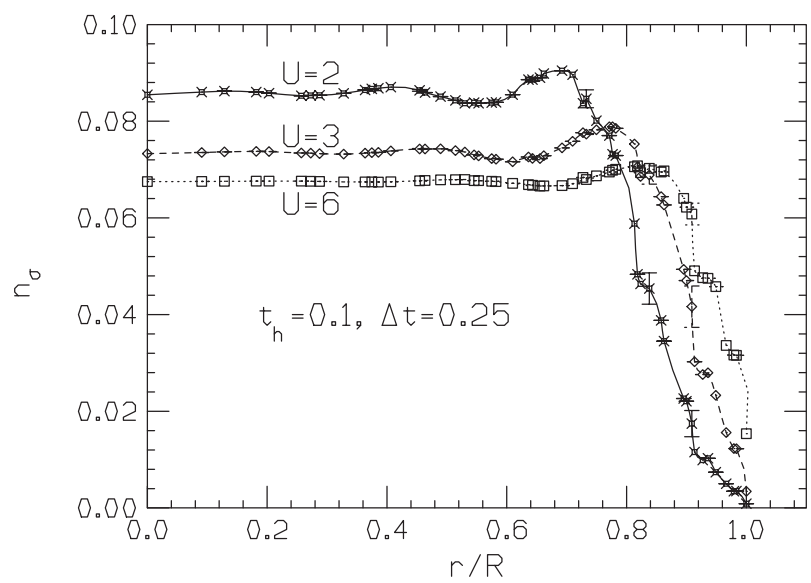

FIG. 6: Effect of Coulomb repulsion. $t_{h}=0.1, \Delta t=0.25$. As the on-site repulsion increases, the charge expulsion decreases and the occupation becomes more uniform.

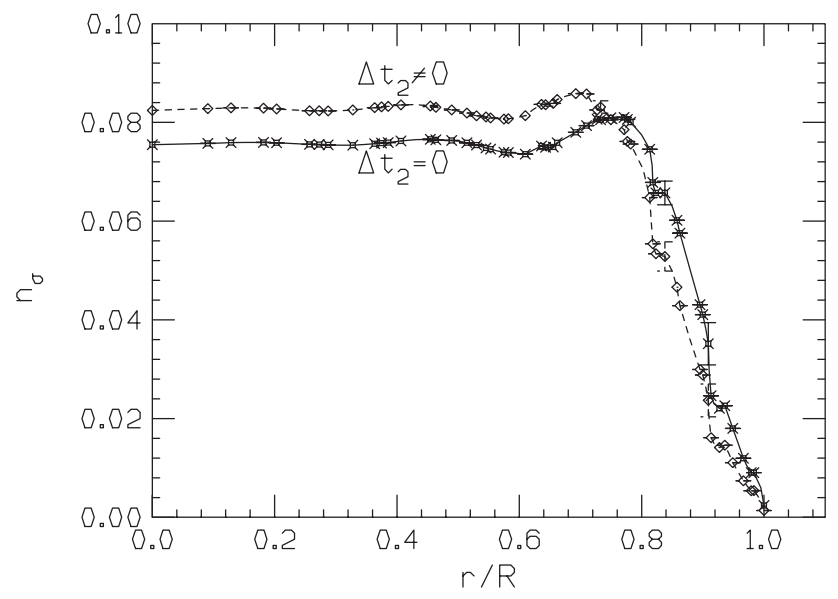

FIG. 7: Effect of non-linear occupation-dependent hopping term $\Delta t_{2}$, Eq. (7c) for the case $\Delta t=0.25$ of Fig. 3 .

increases the charge expulsion tendency relative to the model where this term is omitted (Hubbard model with correlated hopping).

As the correlated hopping amplitude $\Delta t$ increases, and even more so in the presence of $\Delta t_{2}$, the system appears to develop a tendency to phase separation, where holes condense in the interior and the outer region of the cylinder becomes essentially empty of holes. This happens very rapidly as function of the parameters for the finite system under consideration. Examples are shown in Fig. 8. We return to this point in the next section.

The charge expulsion tendency and associated effects described in this and other sections become rapidly weaker when the hole concentration increases. To illustrate this we show in Fig. 9 the charge distribution for the same Hamiltonian parameters as Fig. 8 but average hole occupation per site $n=0.35$ instead of $n=0.126$. It can be seen that the charge expulsion is much smaller. In summary, we have seen in this section that the dy- 


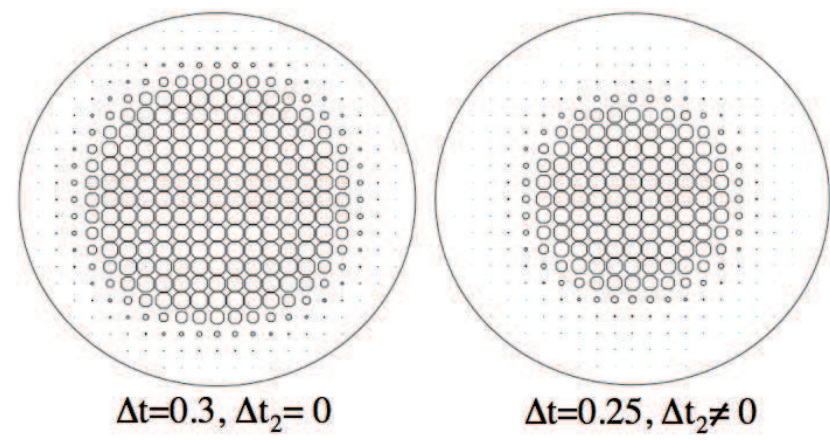

FIG. 8: As the correlated hopping terms increase, the system develops a tendency to phase separation, where essentially all the holes condense to the interior. Parameters are the same as in Fig. 3 except as indicated. The maximum hole occupation per spin is 0.128 and 0.214 for the left and right panel, the average hole occupation per spin is 0.063 .
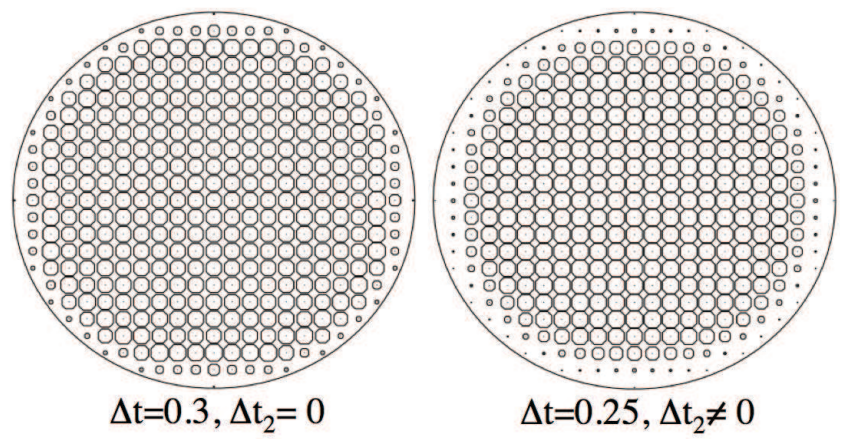

FIG. 9: Hole site occupations for the same parameters as Fig. 8 but average hole occupation per spin 0.175 . The maximum hole occupation per spin is 0.214 for the left panel, 0.232 for the right panel.

namic Hubbard model promotes expulsion of negative charge from the interior to the surface of the system when the band is almost full, and that the charge expulsion physics is associated with kinetic energy lowering, just as in the single atom, Fig. 1. The charge expulsion tendency is largest when the parameter $\Delta t$ is largest, which in turn corresponds to smaller $S$, the overlap of the atomic orbitals when one and two electrons are at the orbital. As discussed earlier, $S$ is smaller when the ionic charge $Z$ is smaller, corresponding to a more negatively charged ion. The fact that the effective Hamiltonian derived from this physics expels more negative charge the more negatively charged the ion is makes of course a lot of sense and can be regarded as an internal consistency check on the validity of the model.

\section{PHASE SEPARATION}

The tendency to charge expulsion in the dynamic Hubbard model can also lead to a thermodynamic instability and ensuing phase separation. The question of phase sep- aration in these models in certain parameter ranges was recently considered by Montorsi and coworkers [40, 41].

Let us consider the correlated hopping model first for simplicity $\left(\Delta t_{2}=0\right)$. The effective hopping amplitude for a hole is $t(n)=t_{h}+n \Delta t$ with $n$ the hole density per site. In the dilute limit, the kinetic energy of a hole is $\epsilon=-z t(n)$, with $z$ the number of nearest neighbors to a site, and it is clear that when $\Delta t$ is much larger than $t_{h}$ putting for example all holes into half the system and thus emptying the other half will double $n$ and hence decrease the kinetic energy per hole as well as the total energy if it is dominated by kinetic energy. This tendency to phase separation will be countered both by Pauli exclusion and by Coulomb repulsion.

Consider a flat density of states for simplicity. The effective bandwidth increases linearly with $n$

$$
D(n)=D_{h}+K n
$$

with $D=2 z t, D_{h}=2 z t_{h}, K=2 z \Delta t$. The density of states per site per spin is given by $g(\epsilon)=1 / D$ and the ground state kinetic energy by

$$
E_{k i n}=\int_{-D / 2}^{\mu} \epsilon g(\epsilon) d \epsilon=\frac{D}{4}\left(n^{2}-2 n\right)
$$

with $\mu=(n-1) D(n) / 2$ the chemical potential for $n$ holes per site. Adding the on-site repulsion in a mean field approximation yields

$$
E_{o}(n)=\frac{D_{h}+n K}{4}\left(n^{2}-2 n\right)+\frac{U}{4} n^{2}
$$

and the system will be unstable towards phase separation into hole-rich and hole-poor regions when the condition

$$
\frac{\partial^{2} E_{0}}{\partial n^{2}}=\frac{U+D_{h}}{2}+K\left(\frac{3}{2} n-1\right)<0
$$

is satisfied, hence

$$
K>\frac{U+D_{h}}{2\left(1-\frac{3}{2} n\right)}
$$

or equivalently

$$
\Delta t>\frac{t_{h}+U /(2 z)}{2\left(1-\frac{3}{2} n\right)} .
$$

For the parameters used in the previous section, e.g. $t_{h}=0.1, n=0.126, U=2$ and $z=4$ appropriate to two dimensions Eq. (21) yields $\Delta t>0.216$. The tendency to phase separation becomes even stronger when the nonlinear term $\Delta t_{2}$ is included. After some simple algebra Eq. (21) is modified to the condition

$$
\Delta t>\frac{t_{h}+U /(2 z)}{2\left(1-\frac{3}{2} n\right)}-\frac{3 \Delta t^{2} n(1-n) /\left(4 t_{h}\right)}{2\left(1-\frac{3}{2} n\right)}
$$

which for the parameters given above yields $\Delta t>0.182$. These estimates are consistent with the numerical results 
shown in the previous section. Note that as $n$ increases larger $\Delta t$ is needed for phase separation.

Note that the instability criterion Eq. (19) appears to be different from the usual criterion

$$
\frac{\partial \mu}{\partial n}<0
$$

if $\mu$ is given by the expression given right after Eq. (17). The reason is that the $\mu$ in Eq. (17) is not the true chemical potential but an effective one. The true chemical potential is modified by contributions from both the Hubbard repulsion and the density dependent hopping terms. For the case with $\Delta t_{2}=0$ it is given by

$$
\begin{gathered}
\mu=\mu_{e f f}+\frac{U n}{2}-\frac{K}{2}<c_{i \sigma}^{\dagger} c_{j \sigma}+h . c .> \\
\mu_{e f f}=\frac{D_{h}+n K}{2}(n-1) \\
<c_{i \sigma}^{\dagger} c_{j \sigma}+\text { h.c. }>=n\left(1-\frac{n}{2}\right)
\end{gathered}
$$

where the expectation value for the bond charge Eq. (24c) follows from Eq. (17). Hence we obtain from Eq. (24)

$$
\frac{\partial \mu}{\partial n}=\frac{U+D_{h}}{2}-K\left(1-\frac{3}{2} n\right)
$$

in agreement with Eq. (19). The instability criteria Eq. (23) or Eq. (19) with the free energy replacing $E_{0}$, are also valid at finite temperature of course.

In a real material in the normal state phase separation will not occur because of the effect of longer-range Coulomb interactions not included in our model Hamiltonian. However this physics will clearly favor charge inhomogeneity, i.e. hole-rich regions that benefit from the lowering of kinetic energy acquired by increasing the hole concentration and thereby broadening the (local) bandwidth, and hole-poor regions where the kinetic energy cost is mitigated by narrowing of the local bandwidth. The shape of these regions will depend on the particular details of the system and merits further investigation. A possible geometry for the hole-rich and holepoor regions could be one-dimensional, i.e. stripes 23. Other geometries like patches are also possible [25, 26]. Such charge inhomogeneities are commonly seen in high $T_{c}$ superconductors [22] where the physics discussed here should be dominant.

\section{CHARGE INHOMOGENEITY}

High $T_{c}$ cuprates show a high tendency to charge inhomogeneity [22, 24 26]. We suggest that a dynamic Hubbard model may be relevant to describe this physics: because kinetic energy dominates the physics of the dynamic Hubbard model, the system will develop charge inhomogeneity at a cost in potential energy if it can thereby

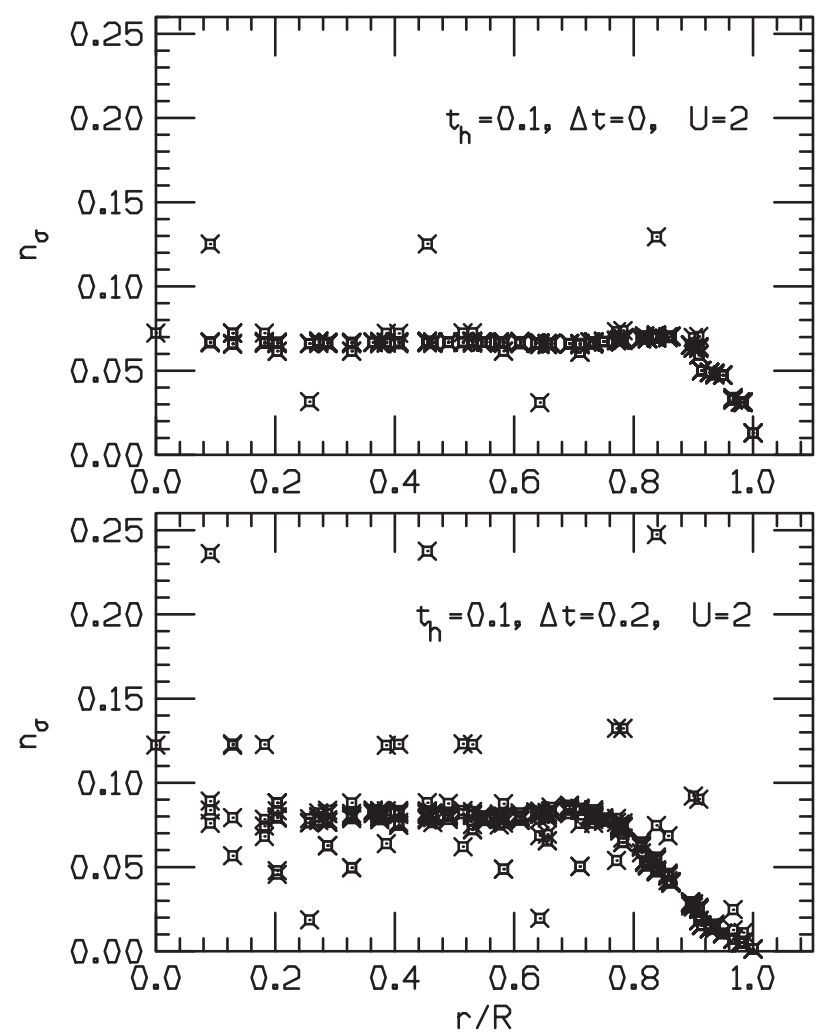

FIG. 10: Hole site occupation per spin in a system of radius $R=11$ with 5 impurities at positions $(-1,0),(2,2),(3,-4),(-$ $5,-5),(-6,7)$ with potential strength $-0.2,+0.2,-0.2,+0.2$, -0.2 respectively. Note the much larger variation in densities generated in the dynamic Hubbard model (lower panel, $\Delta t_{2} \neq$ $0)$ than in the conventional Hubbard model (upper panel). Average hole occupation per site is $n=0.126$.

lower its kinetic energy more, unlike models where the dominant physics is potential (correlation) energy like the conventional Hubbard model.

We assume there are impurities in the system that change the local potential at some sites, and compare the effect of such perturbations for the dynamic and conventional Hubbard models. As an example we take parameters $t_{h}=0.1, U=2$ and consider site impurity potentials of magnitude \pm 0.2 at several sites as indicated in the caption of Fig. 8. For the dynamic Hubbard model we take $\Delta t=0.2, \Delta t_{2}=0.4$, corresponding to $S=0.333$.

Figure 10 shows the effect of these impurities on the charge occupation for the conventional and dynamic models. In the conventional Hubbard model the occupation changes at the site of the impurity potential and only very slightly at neighboring sites. In the dynamic Hubbard model the local occupation change at the impurity site itself is much larger than in the conventional model, and in addition, the occupations change at many other sites in the vicinity of the impurities, as seen in the lower panel of Figure 10. Figure 11 shows the real space distribution of these changes.

Similarly we can consider impurities where the atomic 

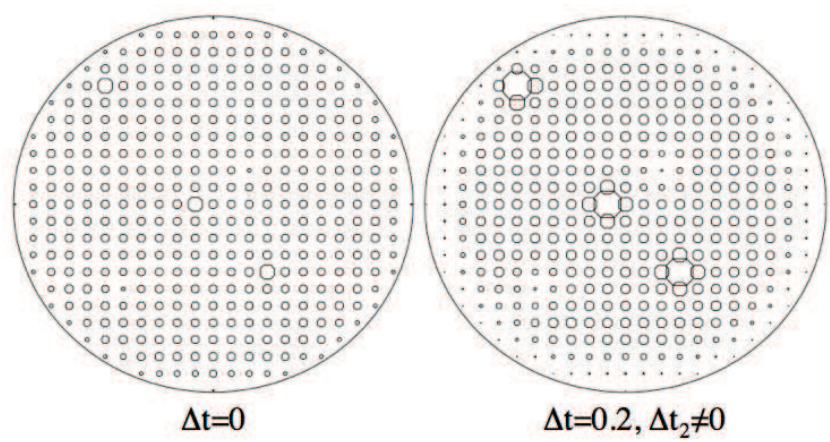

FIG. 11: The site occupations for the case of Fig. 10, with the diameters of the circles proportional to the hole occupation of the sites. Note the 5 impurity sites at positions listed in the caption of Fig. 10 (three with negative potential, hence higher hole concentration) and two with positive potential, hence lower hole concentration. Note that for $\Delta t=0$ only the occupation at the impurity site changes appreciably, while for $\Delta t \neq 0$ an impurity potential of the same strength causes a much larger change of the occupation at the impurity site and occupation change also at the nearest and next nearest neighbor sites.

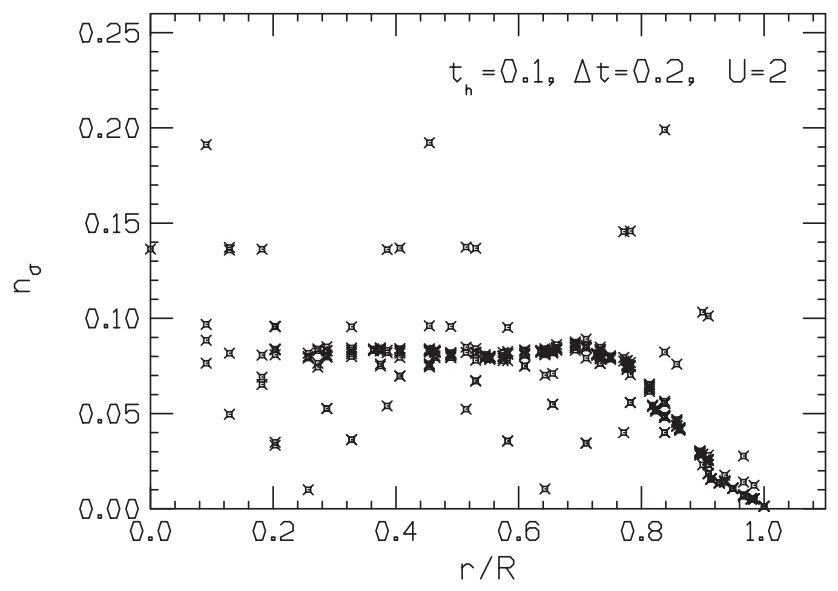

FIG. 12: Hole site occupation per spin in a system of radius $R=11$ with 5 impurities at positions $(-1,0),(2,2),(3,-4),(-5$, $-5),(-6,7)$ with $S$ factor $0.5,0.2,0.5,0.2,0.5$ respectively. All other sites have $S=0.333$. $n=0.126$. Note that the variations in density occur for even more sites than when the local potential is changed, Fig. 10 lower panel, even though the change in occupation at the impurity site itself is smaller.

value of the deformation parameter $S$ is different than in the bulk. This would occur for example by substituting an ion by another ion with different ionic charge, hence different orbital rigidity. For example, substituting $\mathrm{O}^{--}$ by $F^{-}$would make the orbital more rigid and increase $S$ at this site, while substituting $O^{--}$by $N^{---}$would make the orbital more floppy and decrease $S$. Figure 12 shows 5 impurities at the same locations as in Figure 11, with values $S=0.5$ and 0.2 at the impurity sites instead of the bulk value $S=1 / 3$. The larger (smaller) $S$ will increase (decrease) the occupation. Compared to the case
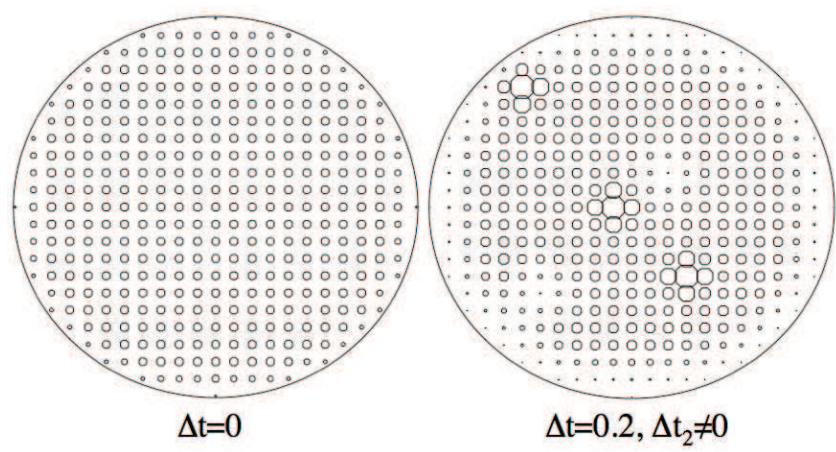

FIG. 13: The site occupations for the case of Fig. 11, with the diameters of the circles proportional to the hole occupation of the sites. Same 5 impurity sites at positions listed in the caption of Fig. 10 (three with larger $S$, hence higher hole concentration) and two with smaller $S$, hence lower hole concentration. Note that the range of sites where the occupation changes is even larger than in Fig. 11, extending to third nearest neighbors. The left panel shows for comparison a uniform system, corresponding to a conventional Hubbard model that does not take into account the deformation of the orbital, hence $S=1$ for all sites.

of Fig. 10, it can be seen that the change in occupation at the impurity site itself is somewhat smaller for these parameters but the changes are larger at neighboring sites and extend to sites farther away. Similarly as in Fig. 11 we show the real space changes in Figure 13, compared to the conventional Hubbard model where no change at all would occur since it is not sensitive to the rigidity of the orbital.

The changes in site energies in the example of Figs. 10 and 11 could occur both if there are substitutional impurities in the conducting plane with different on-site energies, or if there are impurities off the plane that change the local potential in the plane. In contrast, the example of Figs. 12 and 13 would be appropriate to describe only impurities in the plane itself where the electrons conduct.

The reason for this large sensitivity to local perturbations can be understood from the form of the hopping amplitude Eq. (14b). A change in the local occupation will also change the hopping amplitude of a hole between that site and neighboring sites, which in turn will change the occupation of neighboring sites, and so on. Similarly a change in the deformation parameter $S$ at a site will affect the hopping amplitudes between that site and its nearest neighbors, hence the occupation of the site and its neighbors, etc. In that way a local perturbation in the dynamic Hubbard model gets amplified and expanded to its neighboring region, and it is easy to understand how a non-perfect crystal will easily develop patches of charge inhomogeneity in the presence of small perturbations. These inhomogeneities cost potential (electrostatic) energy, but are advantageous in kinetic energy. The conventional Hubbard model does not exhibit this physics. 


\section{GRAIN BOUNDARIES IN HIGH $T_{c}$ CUPRATES}

In this section we argue that dynamic Hubbard models may be relevant to the understanding of properties of grain boundaries in high $T_{c}$ cuprates [42 44] and other materials.

Babcock et al 45] report results of EELS experiments indicating severe hole depletion in YBCO near grain boundaries for large angle grain boundaries, with the hole deficient region extending up to $100 \AA$ or more into the crystal. In contrast, small angle grain boundaries show significantly less hole depletion. It is reasonable to assume that for larger angle grain boundaries there is weaker coupling betwen the grains, and this is confirmed experimentally by measurement of the Josephson critical current across the grain boundary [45]. Furthermore, Babcock et al found that the structural changes associated with the grain boundaries (structural perturbations and cation nonstochiometry) extended only about $5 \AA$ from the grain boundary into the crystal, and that the hole depletion is not associated with particular specimen preparation procedures such as time duration of oxygen annealing and storage conditions. The fact that the hole depletion region extends over a much wider region that associated with structural changes suggests an intrinsic purely electronic origin for the effect. Other workers have found similar results, including Browning et al[46] and Schneider et al 47].

Furthermore and consistent with this picture, it has been found that substituting $Y$ by $C a$ near grain boundaries is an efficient way to prevent the hole depletion phenomenon [48 [50], since $\mathrm{Ca}^{++}$ions will donate fewer electrons to the $\mathrm{CuO}$ planes than the $Y^{+++}$ions they substitute, thus increasing the hole concentration and thereby improving the transport properties across the grain boundary.

Previous theoretical explanations of these effects have implicitly or explicitly assumed that grain boundaries in high $T_{c}$ cuprates have an intrinsic positive charge that leads to band bending and consequently a flow of conduction electrons to the vicinity of the grain boundaries that causes hole depletion 51 53. However, these explanations are directly contradicted by experiments that measure the electric potential at the grain boundary by electron-beam holography [54]. These experiments show unequivocally that the electrostatic potential at the grain boundary is negative with respect to the interior [55 57].

To make sense of the electron holography results, Mannhart suggested 44] that the negative potential at the grain boundary core may cause overdoping of the lower Hubbard band with holes, resulting in an empty band and insulating behavior. However, this explanation would appear to be inconsistent with the experiments of Babcock et al 45] discussed above, as well as with the evidence that hole doping near the grain boundaries by $\mathrm{Ca}$ substituting $Y$ improves the conduction across grain boundaries. Klie et al argue that their calculations [58]
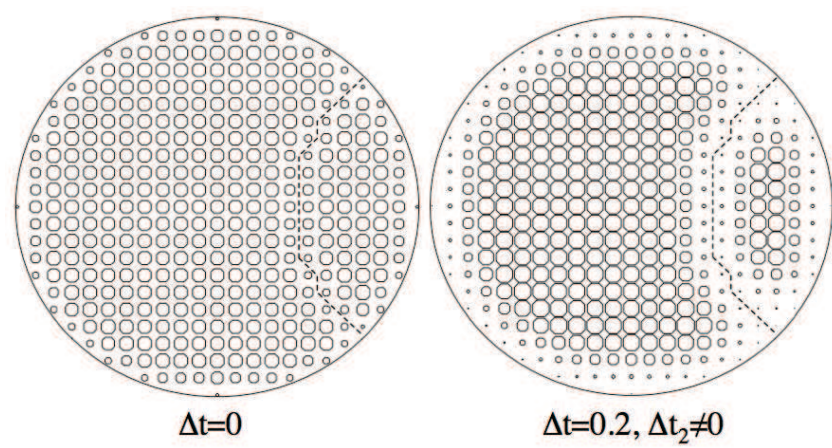

FIG. 14: Effect of a grain boundary, indicated by the dashed line for the conventional and dynamic Hubbard models with $t_{h}=0.1, U=2$. We assume that the hopping amplitude is reduced by a factor 0.3 for sites on opposite sides of the grain boundary. The hole occupation is depleted in the vicinity of the grain boundary in both cases, however the effect is much larger and extends over a wider range for the dynamic Hubbard model than for the conventional one. Temperature is $T=0.02$.
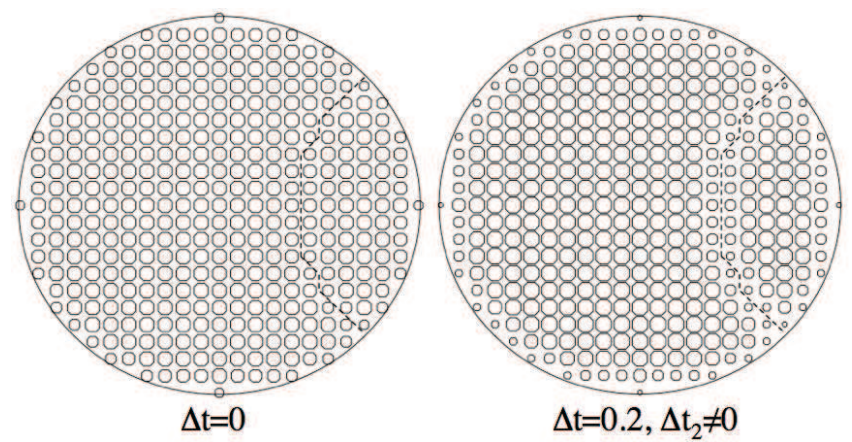

FIG. 15: Same as Fig. 14 with temperature $T=0.1$

support the hole depletion scenario (hence positive potential at the grain boundary) and that this raises question about what potential is measured in the electron holography experiments. However, Koch [59] argues that "it seems to be possible to rule out that this observation is a measurement artifact inherent to the method of electron holography".

These seemingly contradictory observations however are consistent with the physics predicted by the model under consideration here. We model the grain boundary by assuming a smaller hopping amplitude between sites at opposite sides of the grain boundary. A large angle grain boundary would presumably have a more reduced hopping amplitude compared to a small angle grain boundary. Figure 14 shows the charge distribution resulting from our model assuming a $70 \%$ reduction in the hopping amplitude across the grain boundary (denoted by a dashed line), presumably corresponding to a fairly large angle grain boundary with significant increase in the resistance. The hole density in the vicinity of the grain boundary is significantly reduced, driven by lowering of kinetic energy of the system. As a conse- 
quence, the negative charge density becomes larger near the grain boundary, and this would give rise to a negative electric potential at the grain boundary, consistent with the electron holography experiments, and a depleted hole concentration around the grain boundary, consistent with the EELS experiments [45].

As a function of temperature, Schneider et al [47] and Mennema et al 60] find that increasing the temperature leads to a rapid decrease of the grain boundary resistance. We suggests that this results from an increase in the hole concentration near the grain boundary as the temperature is increased. Figure 15 shows that as the temperature is increased the hole density near the grain boundary increases substantially in the dynamic Hubbard model, and as a consequence the conductivity of the region would increases. It would be interesting to measure directly the dependence of hole depletion on temperature by EELS experiments. This has not yet been done to our knowledge.

Recent measurement of properties of grain boundaries in Fe-As superconductors [61] indicate that the behavior is similar to the high $T_{c}$ cuprates. This would not be surprising if the physics of both classes of materials is described by dynamic Hubbard models.

\section{DISCUSSION}

Both the conventional Hubbard model and the dynamic Hubbard model are simplified descriptions of real materials, as are other models used to describe electronic materials such as the periodic Anderson model, the Holstein-Hubbard model, the t-J model, etc. Whether any model contains the physics of interest for particular real materials is in principle an open question. In this paper we have argued that the dynamic Hubbard model, which entails a rather straightforward and natural generalization of the conventional Hubbard model motivated by consideration of the physics of atomic orbitals in real atoms, has interesting properties in some parameter regimes that were not known beforehand and that may have implications for the understanding of properties of real materials.

The new physics of the dynamic Hubbard model is that it allows the electronic orbital to expand when it is doubly occupied. This expansion has associated with it outward motion of negative charge as well as lowering of the electron's kinetic energy at the atomic level, and it is intrinsically not "electron-hole symmetric". This physics is not included in the conventional Hubbard model that assumes that the electronic orbital is infinitely rigid. The key question is not whether this physics exists in real atoms, of course it does[3], the key questions are how large the effect is, what are its consequences, and under which conditions and for which materials is it or is it not relevant for various properties of the materials it aims to describe.

The mathematical treatment of dynamic Hubbard models is not simple, and from the outset we restricted ourselves in this paper to the antiadiabatic limit, i.e. assuming that the energy scale associated with the orbit expansion ( $\omega_{0}$ in Eq. (4)) is sufficiently large than it can be assumed infinite. This brings about the simplification that the high energy degrees of freedom can be eliminated and the Hamiltonian becomes equivalent to the low energy effective Hamiltonian Eq. (7), a Hubbard model with correlated hoppings, linear and nonlinear terms $\Delta t$ and $\Delta t_{2}$. This low energy effective Hamiltonian, together with the quasiparticle weight renormalization described by Eq. (6), describes properties that we believe are relevant to real materials and are not described by the conventional Hubbard model.

In this paper we have shown with specific quantitative examples that the dynamic Hubbard model has a strong tendency to expel negative charge from the interior of the system to the surface, driven by lowering of kinetic energy of the charge carriers. We believe it is truly notable that this property of the model mimics the physics of the single atom that motivated the formulation of the model, even though the kinetic energy lowering and negative charge cloud expansion of the atomic electron is not explicitly included in the site Hamiltonian Eq. (1). It is furthermore notable that the orbital expansion in an ion is larger when the ion is negatively charged, which in the model translates to a larger value of the coupling constant $g$ (smaller $S$, Eq. (6b)), larger $\Delta t / t_{h}$ (Eq. (10)) and consequently larger tendency for the model to expel negative charge. We argue that the fact that the lattice Hamiltonian Eq. (9) describes at a macroscopic length scale the same physics at the atomic scale that motivated the Hamiltonian is a strong indication that the Hamiltonian is relevant for the description of reality.

The effects predicted by this Hamiltonian are largest when the coupling constant $g$ is large, or equivalently when the overlap matrix element $S$ is small, which corresponds to a "soft orbital" that would exist for negatively charged anions, and the effects are also largest when the band is almost full with negative electrons (strong coupling regime). Thus, not surprisingly, more negative charge at the ion or/and in the band yield larger tendency to negative charge expulsion for the entire system. We believe that the Hamiltonian is relevant to describe the physics of materials including high $T_{c}$ cuprates, $\mathrm{Fe}$ pnictides, $\mathrm{Fe}$ chalcogenides, $M g B_{2}$ and $B i S_{2}$-based 62] materials. These materials have negatively charged ions $\left(\mathrm{O}^{--}, \mathrm{As}^{---}, \mathrm{S}^{--}, \mathrm{Se}^{--}, \mathrm{B}^{-}\right)$with soft orbitals, and for most of them, including "electrondoped" cuprates 63 there is experimental evidence for dominant hole transport in the normal state. We suggest that the orbital expansion and contraction of these negative ions depending on their electronic occupation is responsible for many interesting properties of these materials and is described by the dynamic Hubbard model.

We have seen in this paper that the model leads to charge inhomogeneity driven by lowering of kinetic energy, and in extreme cases to phase separation, and that 
it leads to negatively charged grain boundaries and depletion of hole carriers in the vicinity of grain boundaries, properties experimentally observed in many of these materials but not understood using conventional models such as "band bending".

Much of the physics of dynamic Hubbard models for finite $\omega_{0}$ remains to be understood. In fact, the model itself may require substantial modification to account for different values of $\omega_{0}$ for different electronic occupations: the excitation spectrum of the neutral hydrogen atom, $H$, is certainly very different from that of $H^{-}$. In connection with this and going beyond the antiadiabatic limit where only diagonal transitions of the auxiliary boson field are taken into account as in this paper, it is possible that vertical transitions may play a key role in understanding certain properties of systems described by dynamic Hubbard models

In other work we discuss the related facts that the
Hamiltonian Eq. (9) gives rise to pairing of holes and superconductivity when the electronic energy band is close to full, driven by lowering of kinetic energy 64 . We have also proposed elsewhere an alternative electrodynamic description of the superconducting state arising from this model that describes expulsion of negative charge from the interior to the surface 65]. Finally, we have pointed out that in the presence of an external magnetic field negative charge expulsion from the interior to the surface would give rise to magnetic field expulsion from the interior to the surface 66,67 .

\section{Acknowledgments}

The author is grateful to F. Guinea for helpful discussions.
[1] "The Hubbard Model: A Collection of Reprints", ed. by A. Montorsi, World Scientific, Singapore, 1992.

[2] "The Hubbard model: its physics and mathematical physics", ed. by D. Baeriswyl, D.K. Campbell, J.M.P. Carmelo, F. Guinea and E. Louis, NATO ASI Series B Vol. 343, Plenum, New York, 1995.

[3] J.C. Slater, Quantum Theory of Atomic Structure, McGraw-Hill, New York, 1960.

[4] J.E. Hirsch, Phys. Lett. A 134, 451 (1989).

[5] A. Fortunelli and A. Painelli, Chem. Phys. Lett. 214, 402 (1993).

[6] J.E. Hirsch, Physica B 199\&200, 366 (1994).

[7] J.E. Hirsch and S. Tang, Sol. St. Comm. 69, 987 (1989).

[8] J.E. Hirsch, Phys. Rev. B 43, 11400 (1991).

[9] J.E. Hirsch, Phys.Rev. Lett. 87, 206402 (2001).

[10] P. Sun and G. Kotliar, Phys. Rev. B 66, 085120 (2002).

[11] A.S. Moskvin and Y.D. Panov, Phys. Rev. B 68, 125109 (2003).

[12] F. Aryasetiawan et al, Phys. Rev. B 70, 195104 (2004).

[13] L. Arrachea and A.A. Aligia, Physica C 408, 224 (2004).

[14] K. Bouadim et al, Phys. Rev. B 77, 014516 (2008).

[15] G. H. Bach, J.E. Hirsch and F. Marsiglio, Phys. Rev. B 82, 155122 (2010).

[16] G. H. Bach and F. Marsiglio, Jour. Sup. Nov. Mag. 24, 1571 (2011).

[17] G. H. Bach and F. Marsiglio, Phys. Rev. B 85, 155134 (2012).

[18] S. Kaiser et al, arXiv:1211.7017 (2012).

[19] J.E. Hirsch, Phys.Rev. B 65, 184502 (2002).

[20] P. Pincus, Solid St. Comm. 11, 51 (1972).

[21] J.E. Hirsch, Physica C 201, 347 (1992).

[22] A. Bianconi et al, Jour. of Phys. Cond. Matt. 12, 10655 (2000).

[23] S.A. Kivelson et al, Rev. Mod. Phys. 75, 1201 (2003).

[24] E. Dagotto, Science 309, 257 (2005).

[25] S.H. Pan et al, Nature 413, 282 (2001).

[26] Y. Kohsaka et al, Nature Physics 8, 534 (2012).

[27] J.E. Hirsch, in "Polarons and Bipolarons in high Tc Superconductors and Related Materials", ed. by E.K.H. Salje, A.S. Alexandrov and W.Y. Liang, Cambridge Uni- versity Press, Cambridge, 1995, p. 234.

[28] S. Robaszkiewicz , R. Micnas and J. Ranninger, Phys. Rev. B 36, 180 (1987).

[29] J.E. Hirsch, Phys. Rev. B 67, 035103 (2003).

[30] J.E. Hirsch, Phys. Rev. B 47, 5351.

[31] J.E. Hirsch, Phys. Rev. B 62, 14487 (2000); Phys. Rev. B 62, 14998 (2000).

[32] J.D. Mahan, "Many Particle Physics", Third Edition, Plenum, New York, 2000.

[33] J.E. Hirsch, Phys. Rev. B 65, 214510 (2002); Phys. Rev. B 66, 064507(2002).

[34] F. Marsiglio, R. Teshima and J. E. Hirsch, Phys. Rev. B 68, 224507 (2003).

[35] M. Casula et al, Phys. Rev. Lett. 109, 126408 (2012).

[36] D. Pines and P. Nozieres, "The Theory of Quantum Liquids", Vol. I, Addison-Wesley, Redwood, City 1989.

[37] S. Kivelson, W.-P. Su, J. R. Schrieffer, and A. J. Heeger, Phys. Rev. Lett. 58, 1899 (1987).

[38] D. K. Campbell, J. Tinka Gammel, and E. Y. Loh Phys. Rev. B 38, 12043 (1988).

[39] G. Kotliar et al, Rev. Mod. Phys. 78, 865 (2006).

[40] A. Montorsi, Jour. Stat. Mech. (2008) L09001.

[41] A. Anfossi, C. D. E. Boschi and A. Montorsi, Phys. Rev. B 79, 235117 (2003).

[42] S.E. Babcock and J.L. Vargas, Ann. Rev. Mat. Sci 25, 193 (1995).

[43] H. Hilgenkamp and J. Mannhart, Rev. Mod. Phys. 74, 485 (2002).

[44] J. Mannhart, in 'Thin Films and Heterostructures for Oxide Electronics', Springer, 2005, p. 251.

[45] S.E. Babcock et al, Physica C 227, 183 (1994).

[46] N.D. Browning et al, Physica C 212, 185 (1993).

[47] C.W. Schneider et al, Phys. Rev. Lett. 92, 257003 (2004).

[48] A. Schmehl et al, Europhys. Lett. 47, 110 (1999).

[49] G. Hammerl et al, Nature 407, 162 (2000).

[50] G.A. Daniels, A. Gurevich and D.C. Larbalestier, Appl. Phys. Lett. 77, 3251 (2000).

[51] J. Mannhart and H. Hilgenkamp, Supercond. Sci. Technol. 10, 880 (1997).

[52] A. Gurevich and E.A. Pashitskii, Phys. Rev. B 57, 13878 
(1998)

[53] U. Schwingenschlogl and C. Schuster, EPL 77, 37007 (2007).

[54] V. Ravikumar, R.P. Rodrigues and V.P. Dravid, Phys. Rev. Lett. 75, 4063 (1995).

[55] M.A. Schofield, L. Wu and Y. Zhu, Phys. Rev. B 67, 224512 (2003).

[56] M.A. Schofield et al, Phys. Rev. Lett. 92, 195502 (2004).

[57] Ch. Joos et al, Physica C 408-410, 443 (2004).

[58] R.F. Klie et al, Nature 435, 475 (2005).

[59] C.T. Koch, Int. J. Mat. Res. 101, 1 (2010).

[60] S. H. Mennema et al, Phys. Rev. B 71, 094509 (2005).

[61] J.H. Durrell et al, Reports of Progress in Physics 74,
124511 (2011) and references therein.

[62] Y. Mizuguchi et al, J. Phys. Soc. Jpn. 81, 114725 (2012).

[63] W. Jiang et al, Phys. Rev. Lett. 73, 1291 (1994); P. Fournier et al , Phys. Rev. B 56, 14149 (1997); Y. Dagan and R.L. Greene, Phys. Rev. B 76, 024506 (2007).

[64] J.E. Hirsch and F. Marsiglio, Phys. Rev. B 39, 11515 (1989); Phys. Rev. B 62, 15131 (2000).

[65] J.E. Hirsch, Phys.Rev. B 68, 184502 (2003); Phys.Rev. B 69, 214515 (2004).

[66] J.E. Hirsch, Physica Scripta 85, 035704 (2012).

[67] W.A. Newcomb, Ann. Phys. 3, 347 (1958). 